\title{
Massgeschneiderte Fortbildung in spezieller Umgebung
}

\author{
Für das 3. Great Update lädt die Schweizerische Gesellschaft für Allgemeine Innere \\ Medizin (SGIM) am 14. und 15. November 2013 zum dritten Mal in den wunder- \\ schönen Kursaal Interlaken ein. Zahlreiche Referentinnen und Referenten bieten ein \\ spannendes, praxisorientiertes Programm für Haus- und Spitalärzte. Im folgenden \\ Interview geben die Co-Tagungspräsidenten Jonas Rutishauser und Jörg D. Leuppi \\ Auskunft über Konzept und Inhalt der Fortbildungstage.
}

Interview: Lukas Zemp

SGIM-Generalsekretär

Korrespondenz: Lukas Zemp Schweizerische Gesellschaft für Allgemeine Innere Medizin Postfach 422

CH-4008 Basel

Tel. 0612259335

sgim[at]sgim.ch

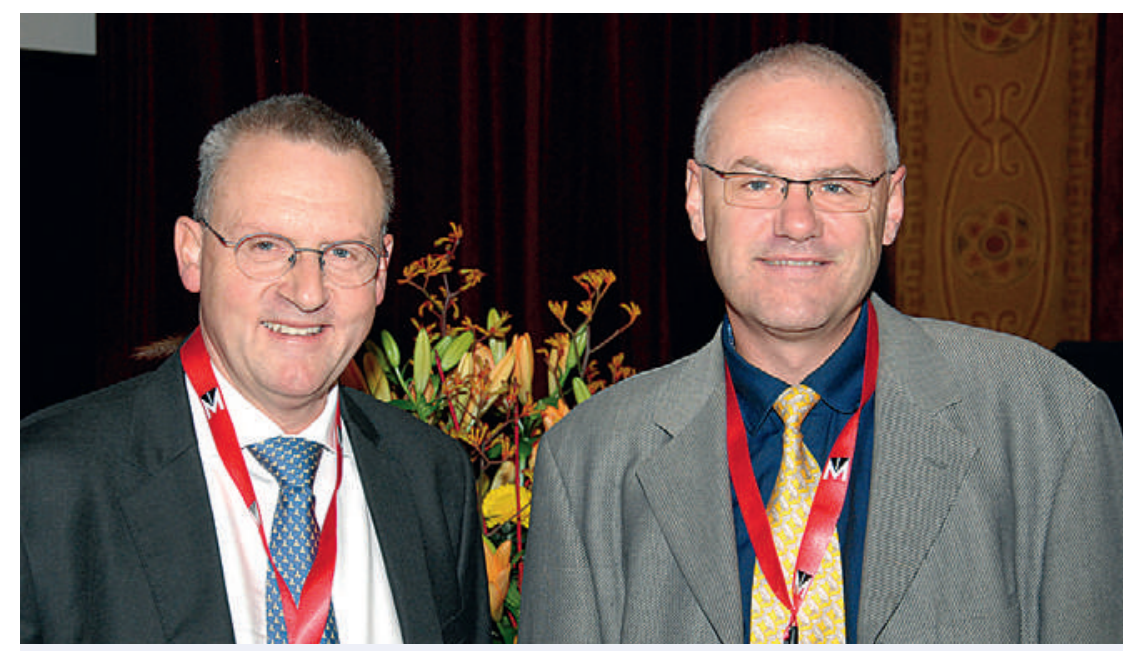

Die beiden Co-Tagungspräsidenten:

Prof. Dr. med. Jörg D. Leuppi (links) und Prof. Dr. med. Jonas Rutishauser. sammlung?

Jonas Rutishauser: Das Great Update ist ein reiner Fortbildungskongress und richtet sich in erster Linie an niedergelassene Grundversorger. Aber auch Kolleginnen und Kollegen in Spitälern, die eine allgemeininternistische Praxistätigkeit ins Auge fassen, profitieren vom dargebotenen Wissen. Zusammen mit der Jahresversammlung, die ja auch wissenschaftliche Akzente setzt, verfügt die SGIM so über eine sinnvolle Kombination zweier attraktiver und sich ergänzender Fortbildungsveranstaltungen.

Jörg D. Leuppi: Dank den erfolgreichen ersten beiden Kongressen hat sich das Great Update bereits fest im SGIM-Fortbildungsprogramm und bei den Fortbildenden, Ausstellern und Sponsoren etabliert. Über die rege Teilnahme aus der Romandie haben wir uns 2011 und 2012 zudem besonders gefreut.
War der Erfolg des SGIM-Great Update von Anfang an voraussehbar? Wie waren die Rückmeldungen zum SGIM-Great Update 2012?

Rutishauser: Natürlich war der Erfolg nicht planbar. Anfänglich musste das SGIM-Great Update zuerst sein eigenes, klares Profil gegenüber der SGIM-Jahresversammlung, dem traditionellen «Klassentreffen der Internisten», entwickeln. Das Great Update dagegen legt mehr Gewicht auf die praktische Umsetzung neuerworbenen medizinischen Wissens.

Die Rückmeldungen zum Great Update 2012 waren durchwegs sehr positiv. Diverse Anregungen haben wir ins diesjährige Programm einfliessen lassen, für das wir zahlreiche Referierende aus Spital und Praxis verpflichten konnten. So können wir mit dem Great Update 2013 wieder ein ausgewogenes Programm anbieten, das sich zielgerichtet an den Ansprüchen der Teilnehmenden orientiert.

Leuppi: Zusammen mit einem kompetenten und motivierten wissenschaftlichen Komitee und dank der Unterstützung eines professionellen Kongressorganisators, der IMK, ist es uns gelungen, den neuen SGIM-Fortbildungsanlass im stark kompetitiven Kongress- und Fortbildungsumfeld erfolgreich zu positionieren. Es ist uns wichtig, das Programm der Veranstaltung kontinuierlich zu optimieren und auf die Bedürfnisse der Teilnehmenden zuzuschneiden. Deshalb wurde die inzwischen bewährte Struktur beibehalten: die Workshops, die den Hauptschwerpunkt bilden, werden von Vorträgen umrahmt, so dass die Fortbildung auch dieses Jahr wieder ein praxisorientiertes und gleichzeitig vielseitiges Programm bietet.

Wie setzt sich das wissenschaftliche Komitee des Great Update 2013 zusammen?

Leuppi: Das wissenschaftliche Komitee des Great Update 2013 besteht aus praktizierenden Kollegen 
und aus Spitalinternisten: Co-Tagungspräsident Prof. Dr. med. Jonas Rutishauser (Bruderholz) und mir als Co-Tagungspräsident (Prof. Dr. Jörg Leuppi, Liestal), sowie Dr. med. Romeo Providoli (Sierre), Dr. med. Sven Streit (Institut für Hausarztmedizin, Bern) und Dr. med. Susanna Stöhr (Luzern).

Was genau dürfen die Teilnehmerinnen und Teilnehmer nun am SGIM-Great Update 2013 in Interlaken erwarten?

Leuppi: Das Great Update ist eine zweisprachige Veranstaltung. Auch dieses Jahr wechseln sich deutsche und französische Beiträge ab. Es stehen unter anderem Referate zu Themen wie Vorhofflimmern, präoperative Abklärungen, Herzinsuffizienz, Bauchschmerz, Schilddrüsenerkrankungen oder Abklärung von Lungenrundherden auf dem Programm.

\section{«Das Great Update dagegen legt mehr Gewicht auf die praktische Umsetzung neuerworbenen medizinischen} Wissens.»

Aktuelle Forumthemen

Diskutieren Sie mit Im Forum präsentieren wir regelmässig brisante Themen aus Politik, Ökonomie und Wissenschaft, die das Schweizer Gesundheitswesen betreffen. Bringen Sie Ihre Meinung ein oder kommentieren Sie die Äusserungen Ihrer Kolleginnen und Kollegen. Das Forum finden Sie unter: www.saez.ch/forum/
Rutishauser: Die Workshops werden von Allgemeininternisten, Kollegen aus internistischen Spezialdisziplinen und Vertretern anderer Fächer moderiert. Hier können Sie zum Beispiel Urinsedimente beurteilen, Wundversorgung oder Spirometrie praktisch üben und Wertvolles zur Reanimation erlernen. Andere Workshop-Themen sind Dyspnoe-Abklärung, Diabetologie mit praktischen Fallbeispielen und
Beurteilung von Thorax-Röntgenbildern. Aber auch Tipps zu praxisrelevanten Apps und Hinweise zur kritischen Lektüre von wissenschaftlichen Papers werden Ihnen geboten. Neu dieses Jahr sind auch die «Hot Topics» als Beiträge der Co-Tagungspräsidenten.

\section{«Die Workshops, die den Haupt- schwerpunkt bilden, werden von Vorträgen umrahmt.»}

Warum findet das SGIM-Great Update 2013 wiederum in Interlaken statt?

Leuppi: Wir werden auch dieses Great Update 2013 im zentral und wunderschön gelegenen Interlaken durchführen, denn das Kongresszentrum Kursaal bietet für unsere Fortbildung den perfekten und persönlichen Rahmen. Die Rückmeldungen der Teilnehmenden haben uns dies bestätigt.

Rutishauser: Wie letztes Jahr werden wir viele Möglichkeiten zum Gespräch zwischen Kolleginnen und Kollegen aus Praxis und Spital haben. Und «last but not least» freuen wir uns auf den Festabend in der stilvollen Brasserie des Hotels Victoria-Jungfrau in Interlaken! Sie sind herzlich nach Interlaken eingeladen.

Zusätzliche Informationen sind $\mathrm{zu}$ finden unter http://kongress2.imk.ch/SGIM2013/Intro oder www.sgim.ch

\section{Sie lesen gerade eine Zeitschrift des Schweizerischen Ärzteverlags EMH ...}

\section{Wussten Sie schon,}

- dass EMH ein Gemeinschaftsunternehmen der Verbindung der Schweizer Ärztinnen und Ärzte FMH und der Schwabe AG ist, dem mit Gründung 1488 ältesten Druck- und Verlagshaus der Welt?

- dass EMH mit insgesamt zehn Fachzeitschriften, einem umfangreichen Online-Angebot sowie einem wachsenden Buchprogramm der führende Verlag für medizinische Zeitschriften in der Schweiz ist?

v dass sämtliche bei EMH erscheinenden Zeitschriften offizielle Publikationsorgane der jeweils zuständigen medizinischen Fachorganisationen sind?

Bei EMH werden Publikationen von Ärztinnen und Ärzten für Ärztinnen und Ärzte herausgegeben.

Die unabhängigen Redaktionen mit namhaften Fachpersonen aus der medizinischen Praxis gewährleisten die hohe Qualität und Relevanz der Beiträge, die das gesamte Spektrum der medizinischen Diskussion abdecken. DieThemenwahl orientiert sich nicht daran, wo Werbegelder der Industrie vorliegen, sondern spiegelt die vielfältigen Interessen der Ärzteschaft sowie der Fachorganisationen wider.

Wenn Sie mehr über EMH wissen möchten, finden Sie unter www.emh.ch weitere Informationen sowie die Online-Ausgaben unserer Zeitschriften mit komfortabler Volltext-Suche.

Gerne stehen wir Ihnen für Ihre Fragen auch persönlich zur Verfügung:

EMH Schweizerischer Ärzteverlag AG

Farnsburgerstrasse 8

CH-4132 Muttenz

Marketing und Kommunikation

Tel. +41 (0)614678506 / Fax +41(0)614678556 marketing@emh.ch 